\title{
Study on Gas Leakage Detection and Pressure Difference Identification by Asymmetric Differential Pressure Method
}

\section{Yan Shi}

Beihang University

Jia-Qi Chang

Beihang University

Yi-Xuan Wang ( $\triangle$ magic_wyx@163.com )

Beihang University https://orcid.org/0000-0003-3079-5572

Xue-Lin Zhao

the forth medical center of PLA general hospital

Qing-Zhen Zhang

Beihang University

Li-Man Yang

Beihang University

\section{Research Article}

Keywords: Leakage Detection, System Identification , Asymmetric Tank , Pneumatics, Measurement, Flow Characteristics

Posted Date: November 29th, 2021

DOI: https://doi.org/10.21203/rs.3.rs-1062489/v1

License: (c) (i) This work is licensed under a Creative Commons Attribution 4.0 International License.

Read Full License 


\section{Title page}

\section{Study on Gas Leakage Detection and Pressure Difference Identification by Asymmetric Differential} Pressure Method

Yan Shi, born in 1981, is currently a professor at School of Automation Science and Electrical Engineering, Beihang University, China. E-mail: shiyan@buaa.edu.cn

Jia-Qi Chang, born in 1996, is currently a PhD candidate at School of Automation Science and Electrical Engineering, Beihang University, China.

E-mail: chang_jiaqi@buaa.edu.cn

Yi-Xuan Wang, born in 1989, is currently a lecturer at School of Automation Science and Electrical Engineering, Beihang University, China.

E-mail: magic_wyx@163.com

Xue-Lin Zhao, works currently at Department of Musculoskeletal tumor, Senior Department of Orthopedics, the Fourth Medical Center of PLA General Hospital, China.

E-mail: zxl_199013@163.com

Qing-Zhen Zhang, is currently an associate professor at School of Automation Science and Electrical Engineering, Beihang University, China.

E-mail: zhangqz@buaa.edu.cn

Li-Man Yang, is currently an associate professor at School of Automation Science and Electrical Engineering, Beihang University, China.

E-mail: ylm@buaa.edu.cn

Corresponding author: Yi-Xuan Wang E-mail: magic_wyx@163.com 


\title{
Study on Gas Leakage Detection and Pressure Difference Identification by Asymmetric Differential Pressure Method
}

\author{
Yan Shi ${ }^{1,+} \cdot$ Jia-Qi Chang ${ }^{1} \bullet$ Yi-Xuan Wang ${ }^{1, *} \cdot$ Xue-Lin Zhao ${ }^{2,+} \cdot$ Qing-Zhen Zhang ${ }^{1} \cdot$ Li-Man Yang ${ }^{1}$
}

\begin{abstract}
As the most common actuator in the pneumatic system, the excellent air tightness of the tank is the key to meet the use requirements of automatic equipment. This paper introduces the common air tightness detection contents, and models the inflation and detection process of the differential pressure method. In order to break away from the restriction on the detection efficiency caused by the asynchronous temperature recovery of the two chambers in the asymmetric differential pressure method, the differential calculation of directly detected pressure difference is replaced by the pressure difference substitute formula. The influence of various parameters in the fitting formula is analyzed by simulation, and the effectiveness of this method is verified by experiments.
\end{abstract}

Keywords: Leakage Detection - System Identification • Asymmetric Tank • Pneumatics • Measurement • Flow Characteristics

\section{Introduction}

The research on air tightness is of great significance for servo control [1], pneumatic spring [2], surgical equipment [3], semiconductor manufacturing [4] and so on. Pneumatic servo control is to collect the flow information of some positions in the pneumatic mechanical coupling system, so as to accurately control the changes such as position, speed and acceleration of the controlled body. The pneumatic spring is filled with compressed air in a sealed container, and its elastic effect is realized by using the compressibility

*Correspondence: magic_wyx@163.com

1 School of Automation Science and Electrical Engineering, Beihang University, Beijing 100191, China

2 Department of Musculoskeletal tumor, Senior Department of Orthopedics, the Fourth Medical Center of PLA General Hospital, China. of the gas. These systems need different sealing degrees. When leakage occurs, the output force or output displacement of pneumatic servo control changes greatly, the pneumatic spring cannot meet the elastic requirements, and the surgical equipment cannot reach the appropriate position of patients, which has a great impact on production and life. Therefore, the research on air tightness is a key research in the pneumatic field.

The existing air tightness research includes bubbling method, acoustic emission detection method, pressure detection method and so on. Ultrasonic method is a kind of acoustic emission detection method, which has a lot of research and improvement. Wang proposed that in order to obtain more accurate leakage location information and determine the location of leakage holes in three-dimensional space, an ultrasonic leakage location method based on multi algorithm data fusion is proposed [5], and an ultrasonic method based on data fusion and neural network is proposed to identify the leakage state of the measured object [6]. Piazzetta studied two detection methods to analyze the acoustic data generated by gas leakage based on active arrangement and ultrasonic transducer measurement, and the ultrasonic transmitter that placed in the pipeline to detect fluid from the inside of the container [7]. However, the measurement of ultrasonic method is greatly affected by the environment, and the external air flow disturbance is easy to interfere with the ultrasonic detection. When the measured part is placed in a harsh environment, it is difficult to accurately judge the occurrence and degree of leakage by this method. At the same time, different measuring people, different measuring machines and different measuring positions or angles might also have different measurement results.

Differential pressure method and direct pressure method belong to pressure detection method, and the difference is whether to introduce standard chamber. In direct pressure 
detection, it is not advisable to sacrifice measurement time for measurement accuracy, which has become a bottleneck to improve production efficiency. Differential pressure detection method has become an important means of rapid and accurate air tightness detection, but this method also has some problems, such as relatively long equilibrium time.

The air tightness detection method of differential pressure method mainly refers to the air pressure comparison between the master cavity and the tested cavity through a specific structure. If the air pressure of the master cavity and the tested cavity is balanced, it indicates that the tested body is in a good air tightness state. If there is a pressure difference between the master cavity and the tested cavity, it indicates that the tested cavity has leakage. Compared with other traditional air tightness detection technologies, differential pressure air tightness detection technology has strong automatic detection performance, and the operation requirements of differential pressure air tightness detection technology are relatively simple, which is very suitable for the detection environment with simple equipment requirements.

This method that judge whether there is leakage in the chamber by measuring the differential pressure between two cavity needs to meet that the materials, volumes and other properties of the two chambers are exactly the same, so that the temperature imbalance and environmental interference can be completely eliminated, and the differential pressure between the two chambers is completely caused by leakage during the measurement process. In the actual differential pressure detection process, some scholars have proposed improvement on the two cavities on unequal completely symmetric structure.

Wang studied the differential pressure method, filled the sealed master tank and the tested tank to a certain pressure at the same time, and then closed each other. If there is air leakage in the measured tank, the air leakage can be detected from the different pressures between the two tanks, and the effects of temperature, thermal conductivity, container volume and other parameters on the differential pressure effect can be obtained through simulation [8]. Daniels proposed a new method to quantify the leakage rate of silicon elastomer test pieces for aerospace, as shown in the Figure 1. By adding the differential pressure control system, he improved the standard pressure attenuation method and added the mass point leakage rate analysis. Compared with the results of the standard method, the results calculated by the new method have significantly lower measurement uncertainty [9].

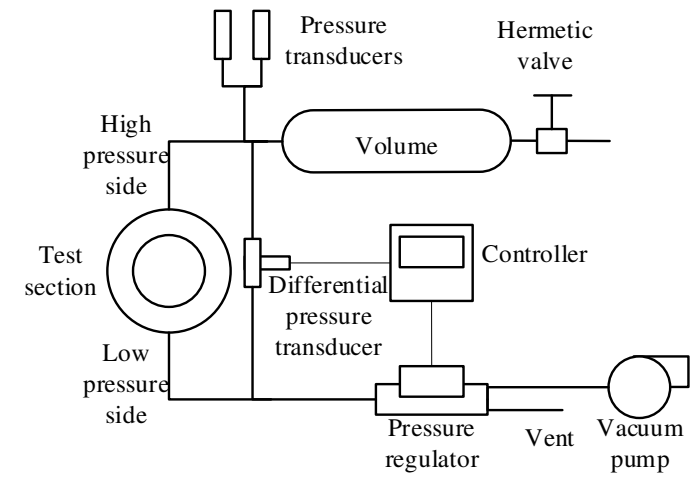

Figure 1 Sealing detection device designed by Daniels

Kagawa et al. built the test-bed shown in the Figure 2 to verify the impact of temperature recovery imbalance on the accuracy and detection ability of the leak detector. When the required minimum balance time is not satisfied, the differential pressure is due to the temperature difference between the two tanks. The longer the balance time is, the smaller the pressure difference between the master tank and the tested tank due to the small difference of temperature balance parameters. Therefore, it is necessary to set the balance time correctly to obtain the maximum performance of the leak detector [10]. Then they discussed temperature recovery and its imbalance due to small heat transfer differences. Because it is difficult to determine the required temperature recovery time through experiments, this study studies the relationship between leakage and thermal time constant, and proposes to use this relationship to estimate the required temperature recovery time [11]. After that, by studying the relationship between temperature recovery time and theoretical thermal time constant under different test volumes and applied pressure, a method for accurately predicting the minimum temperature recovery time considering the repeatability and accuracy of leak detector is proposed, and an air leak detector based on differential pressure is developed [12]. Based on this, they found that, different test pressures and volumes require different temperature recovery times to obtain the expected repeatability and accuracy. The higher the test pressure, the larger the volume, and the longer the temperature recovery time required to obtain the expected repeatability and accuracy [13]. 


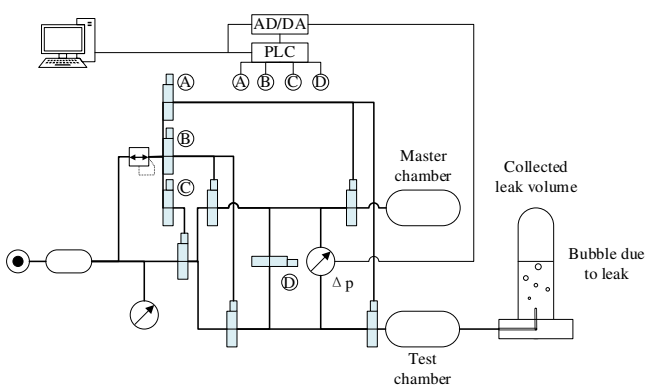

Figure 2 Sealing detection device designed by Kagawa

In addition, in the research of pipeline leakage, Feng proposed a leakage detection and location method based on double sensor pressure gradient method. An example is given to illustrate the industrial application in long-distance oil pipeline, but this method only uses the measured values at the end of the pipeline to detect and locate the leakage in the oil pipeline [14]. Chen has developed a dynamic pressure sensor which can be used to collect leakage signals of long-distance oil and gas pipelines [15].

As the most common actuator in pneumatic system, the excellent air tightness of tank is not only the key to meet the use requirements of automation equipment, but also plays an important role in energy saving and production safety. With the improvement of quality requirements, the detection standard of cylinder is becoming more and more strict. The differential pressure detection method can replace the manual detection and effectively ensure the detection accuracy, but it takes a long time to balance the process to ensure the temperature recovery of the master chamber and the tested chamber, which seriously reduces the detection efficiency. It is of great practical significance to carry out the rapid air tightness detection of tank. Based on the pressure difference method, this paper puts forward some improvement suggestions, establishes the pressure difference simplified model of asymmetric cavity, analyzes the influence of different simplified parameters on the pressure difference results, and verifies that the model has accurate measurement accuracy and short detection time through experiments.

\section{Methods of Asymmetric Differential Pressure Method}

\subsection{Detection Steps of Differential Pressure Method}

The structure diagram of differential pressure detection method is shown in the Figure 3. Its essence is to take the pressure difference between the standard sample arranged on both sides of the sensor and the tested workpiece as the basis for judging the sealing performance of the workpiece, and the pressure difference is caused by the leakage of the workpiece. If the detected workpiece does not leak, the differential pressure sensor will maintain a balanced state. If the detected workpiece leaks, the air pressure in the workpiece measurement chamber will change, causing the differential pressure sensor to lose balance.

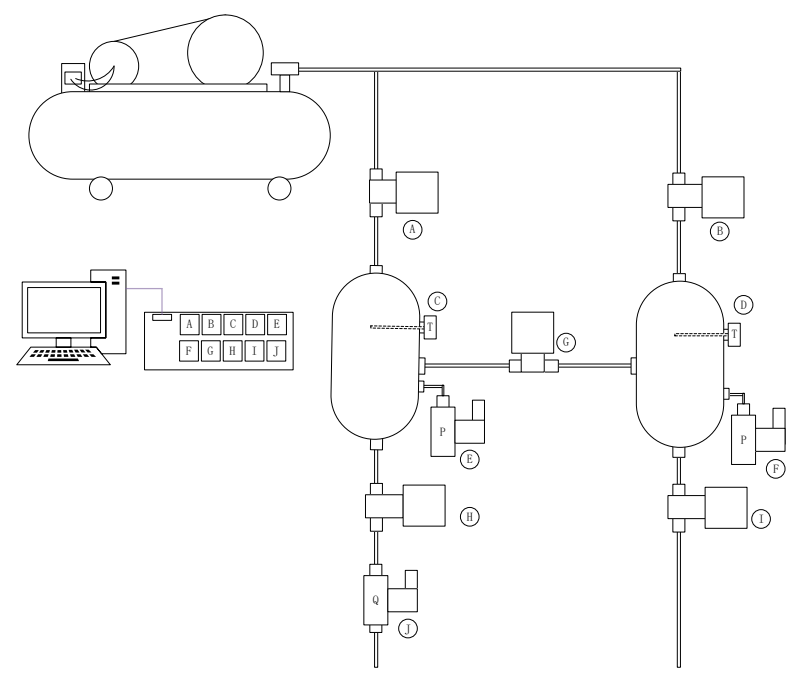

Figure 3 Schematic diagram of leakage measurement by differential pressure method

The specific test steps are as follows:

(1) Inflate both chambers at the same time: Open the pump, valve 1 and valve 2 to keep the pressure filled into the cavity consistent as the standard pressure of the two tanks respectively.

(2) Balance the pressure of the two air tanks: Close valve 1 and valve 2 and open valve 3 to balance the pressure in the master tank and tested tank.

(3) Detect: Read the change of the pressure difference at both ends of the differential pressure sensor, which is caused by the leakage of the tested tank.

(4) Judgment: judge the read pressure difference according to the preset value to determine whether the tightness of the tested tank is qualified.

Therefore, the action sequence of valves 1,2 and 3 is shown in the Figure 4. 


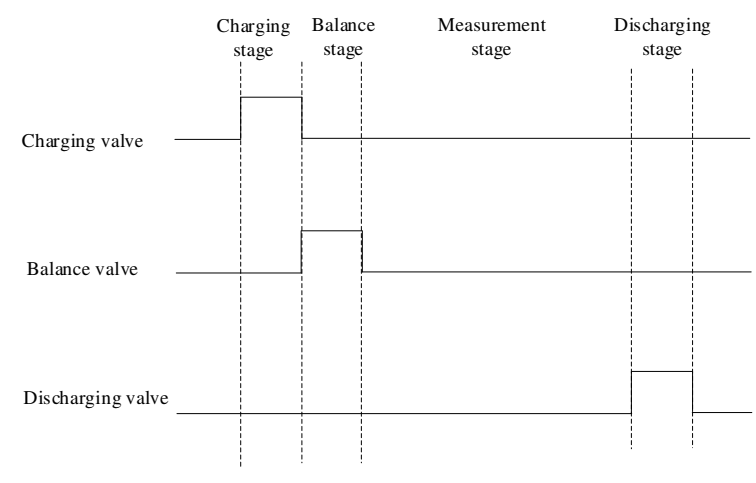

Figure 4 Action sequence of pneumatic valve

For each charging and testing, the pressure of the two chambers should have the trend as follows. Firstly, a large amount of air is rapidly poured into the air tank during charging to increase the internal pressure, until the charging is stopped. In the balance and measurement stage, if the air tank is well sealed, the two chambers affect by cooling on the air tank and reduce the thermal movement of internal air molecules, leading to the reduce of the pressure in the gas tank. If there is leakage in the gas tank, the total amount of air in the gas tank will be reduced and the pressure in the gas tank will be reduced after some air is leaked. In the discharging stage, it can be regarded as a sharp increase in air leakage and a rapid decrease in the pressure in the gas tank.

\subsection{Charging Process and Measurement Process}

During charging, the gas in the master chamber is regarded as an ideal gas, and the pressure change of the master chamber is determined by the ideal gas state equation:

$$
\frac{d P_{m}}{d t}=\frac{P_{m}}{T_{m}} \frac{d T_{m}}{d t}+\frac{R T_{m}}{V_{m}} G_{i m}
$$

Where, $P_{m}$ represents the pressure in the master tank, $T_{m}$ represents the temperature in the master tank, $V_{m}$ represents the volume of the master tank, $G_{i m}$ represents the charging flow rate of the master tank.

For the tested chamber, equation (2) can be obtained according to the energy conservation and the ideal gas state equation. The difference is that there is also mass exchange $G_{e w}$ caused by the leakage of the chamber during charging.

$$
\frac{d P_{w}}{d t}=\frac{P_{w}}{T_{w}} \frac{d T_{w}}{d t}+\frac{R T_{w}}{V_{w}}\left(G_{i w}-G_{e w}\right),
$$

Where, $P_{w}$ represents the pressure in the tested tank, $T_{w}$ represents the temperature in the tested tank, $V_{w}$ represents the volume of the tested tank, $G_{i w}$ represents the charging flow rate of the tested tank.

Considering the leakage mass flow is more less than charging mass flow, the influence caused by leakage can be ignored in the charging stage, so equations (1) and (2) can be simplified into the following equations.

$$
\frac{d P_{w}}{d t}=\frac{P_{w}}{T_{w}} \frac{d T_{w}}{d t}+\frac{R T_{w}}{V_{w}} G_{i w}
$$

During the measurement, the air source interrupts the charging to the tested chamber and the master chamber, and the two chambers are isolated from each other. At this stage, the master cavity only has heat exchange with the environment, while the tested cavity has heat exchange with the environment, and the quality is reduced due to leakage.

For the master cavity, let $G_{i m}=0$ in charging equation (1), the following equation of state for the measurement process can be obtained as shown in equation (4).

$$
\frac{d P_{m}}{d t}=\frac{P_{m}}{T_{m}} \frac{d T_{m}}{d t}
$$

For the tested cavity, let $G_{i w}=0$ in charging equation (2), the following equation of state for the measurement process can be obtained as shown in equation (5).

$$
\frac{d P_{w}}{d t}=\frac{P_{w}}{T_{w}} \frac{d T_{w}}{d t}-\frac{R T_{w}}{V_{w}} G_{e w}
$$

According to equations (4) and (5), the relationship between differential pressure and leakage can be obtained, as shown in equation (6).

$$
\frac{d \Delta P}{d t}=\frac{d P_{w}}{d t}-\frac{d P_{m}}{d t}=\frac{P_{w}}{T_{w}} \frac{d T_{w}}{d t}-\frac{R T_{w}}{V_{w}} G_{e w}-\frac{P_{m}}{T_{m}} \frac{d T_{m}}{d t},
$$

Theoretically, when the equilibrium time is long enough, the differential of $T_{w}$ and $T_{m}$ is equal to 0 , the above equation (6) can be simplified as follows:

$$
\frac{d \Delta P}{d t}=-\frac{R T_{w}}{V_{w}} G_{e w}
$$

That is,

$$
G_{e w}=-\frac{V_{w} d \Delta P}{R T_{w} d t}
$$

\subsection{Pressure Difference Substitute Formula}

In the actual measurement process, because the pressure measurement inevitably produces measurement fluctuations, resulting in large fluctuations in the differential of the pressure, affecting the measurement results and prolonging 
the measurement time, this section analyzes the substitute formula of the pressure difference from the perspective of mechanism, and calculates the leakage through the substitute formula, which can get the results quickly and accurately.

The differential pressure between the tested chamber and the master chamber satisfies the equation,

$$
\Delta P=P_{w}-P_{m},
$$

In order to solve the expression of $\Delta P$, it can be completed in two steps, that is, first analyze the differential pressure of leak free tank, and then superimpose the leakage differential pressure. The following expression is adopted:

$$
\Delta P=\Delta P_{T}+\Delta P_{L}
$$

When the tank is qualified, $G_{e w}=0$. Then bring it in formulas (9) and (10), as shown in equation (11).

$$
\frac{d P_{w}}{d t}=\frac{R h_{w} S_{w}\left(T_{a}-T_{w}\right)}{C_{v} V_{w}},
$$

Considering the ideal gas equation of state $P_{w}=\rho_{w} R T_{w}$. After replacing $T_{w}$, bring it into formula (11) and sort it out as follows,

$$
\frac{d P_{w}}{d t}+\frac{h_{w} S_{w}}{C_{v} V_{w} \rho_{w}} P_{w}-\frac{R h_{w} S_{w}}{C_{v} V_{w}} T_{a}=0,
$$

Where, $\rho_{w}$ is the density of compressed air in the tested chamber. Formula (12) is the non-homogeneous first-order differential equation of the tested cavity pressure, and the solution of the equation finally solved is,

$$
P_{w}=\rho_{w} R\left(T_{0}-T_{a}\right) e^{-\frac{h_{w} S_{w}}{C_{v} V_{w} \rho_{w}} t}+\rho_{w} R T_{a}
$$

After replacing each constant parameter with a parameter such as (14), it obtains $P_{w}=A e^{-B t}+C$, where the boundary condition is $P_{w}(0)=\rho_{w} R T_{0}$.

$$
A=\rho_{w} R\left(T_{0}-T_{a}\right), B=\frac{h_{w} S_{w}}{C_{v} V_{w} \rho_{w}}, C=\rho_{w} R T_{a},
$$

Where $T_{0}$ is the temperature of the tested chamber when the valve 2 is closed, which is the starting temperature of the measurement. $T_{a}$ is the ambient temperature, which is the final temperature of the tested cavity. Then the two temperatures meet the following relationship,

$$
P_{s}=\rho_{w} R T_{0},
$$

$$
P_{\infty}=\rho_{w} R T_{a}
$$

The pressure changes of the tested chamber is the difference between the current pressure value and the initial pressure value, and the initial pressure is the value $P_{S}$ at the end of inflation balance. By introducing equation (13) into equation (9), the following expression can be obtained,

$$
\Delta P_{T}=\rho_{w} R\left(T_{0}-T_{a}\right) e^{-\frac{h_{w} S_{w}}{C_{v} V_{w} \rho_{w}} t}+\rho_{w} R T_{a}-P_{s},
$$

The initial pressure and initial temperature satisfy the ideal gas equation of state,

$$
\Delta P_{T}=\rho_{w} R\left(T_{0}-T_{a}\right) e^{-\frac{h_{w} S_{w}}{C_{v} V_{w} \rho_{w}} t}-\rho_{w} R\left(T_{0}-T_{a}\right),
$$

After replacing the temperature value with (15) and (16), it could be deduced that,

$$
\Delta P_{T}=\left(P_{S}-P_{\infty}\right) e^{-\frac{h_{w} S_{w}}{C_{v} V_{w} \rho_{w}} t}-\left(P_{s}-P_{\infty}\right),
$$

Therefore, if the tank has no leakage, the differential pressure after sealing should be as (20), which is the form of exponential function with constant parameter.

$$
\Delta P_{T}=\alpha e^{-\beta t}-\alpha,
$$

Where,

$$
\begin{gathered}
\alpha=P_{s}-P_{\infty}=\rho_{w} R\left(T_{0}-T_{a}\right), \\
\beta=\frac{h_{w} S_{w}}{C_{v} V_{w} \rho_{w}},
\end{gathered}
$$

Considering that the leakage mass flow $G_{e w}$ is very little and has slight impact on the cavity pressure, it can be approximately regarded as a fixed value, and the change of differential pressure caused by leakage is linear and meets the following requirements,

$$
\Delta P_{L}=-\frac{R \theta_{w} G_{e w}}{V_{w}} t
$$

By superposition of equations (20) and (23), the expression of the variation of differential pressure with time under leakage can be obtained, as shown in equation (24), in which the linear parameter is the differential pressure component caused by leakage and the exponential parameter is the differential pressure caused by temperature 
recovery.

$$
\Delta P=\Delta P_{T}+\Delta P_{L}=\alpha e^{-\beta t}-\lambda t-\alpha,
$$

where, $\lambda=\frac{R T_{w} G_{e w}}{V_{w}}$.

Thus, the parameters can be identified according to the collected pressure value for leakage calculation, so as to avoid the influence of large pressure differential on the measurement results.

\section{Simulation of Asymmetric Differential Pressure Method}

In order to study the influence of various parameters in the pressure difference substitute formula on the leakage calculation results, some simulations are done in MATLAB software. In formula (24), when $\alpha$ is set to $1 \mathrm{kPa}$ and $\lambda$ is 0 , the simulation results of $\Delta p$ and time different from $\beta$ are shown in the Figure 5.

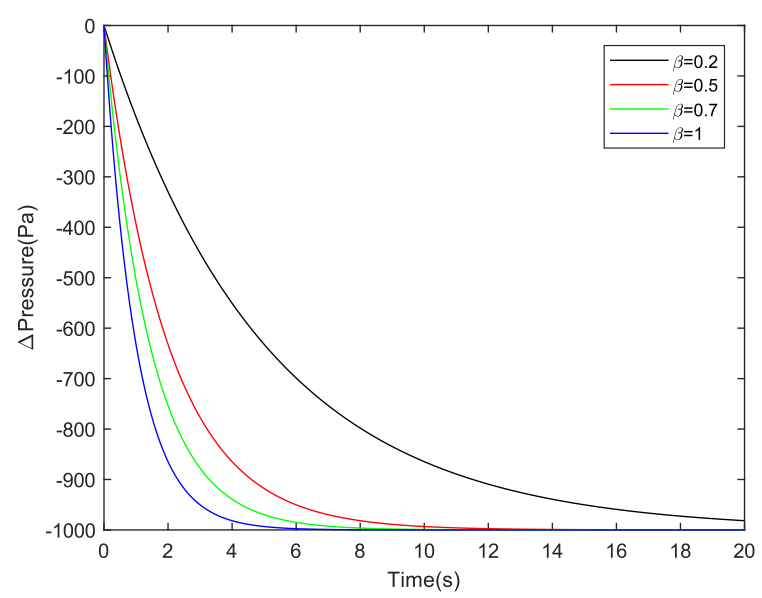

Figure 5 Relationship of $\beta$ and $\Delta p$ without leakage

As can be seen from the Figure 5, with the growth of $\beta$, $\Delta p$ decreases faster and the stabilization time is shorter, indicating that the heat exchange capacity of the tested cavity is enhanced. The pressure difference drops sharply before $10 \mathrm{~s}$, and finally reaches the stable value of $-1000 \mathrm{~Pa}$ for different $\beta$ values. According to (22), the larger heat transfer rate $\mathrm{h}_{\mathrm{w}}$ and heat dissipation area $S_{w}$ of tank, the larger the $\beta$, which means the stronger the heat exchange capacity. After setting the heat balance time constant as $t_{\mathrm{h}}$, which represents the time required for the internal energy of the air in the tank to be transferred to the absolute zero environment, there are the following relationships,

$$
T_{h}=\frac{1}{\beta}=\frac{C_{v} V_{w} \rho_{w}}{h_{w} S_{w}}
$$

It can be seen from the formula that the heat balance time is directly proportional to the volume and inversely proportional to the heat transfer material coefficient and heat transfer area. When the measured volume is large, the heat balance time is obviously prolonged. When the material with excellent heat transfer material is used and the heat transfer area is increased, the heat balance time will also be reduced. When the gas tank is determined, the balance time and $\beta$ value is independent of other parameters.

In order to explore the relationship between $\beta$ and $\Delta p$ in case of leakage, in (24), when $\alpha$ is $1 \mathrm{kPa}$ and $\lambda$ is $1 \mathrm{kPa}$, the simulation results of $\Delta p$ and time of different $\beta$ are shown in the Figure 6 . It can be seen that $\beta$ has little relationship with the leakage representative parameter $\lambda$. At the beginning of measurement, the pressure drops with large $\beta$ is larger than that with small $\beta$. However, the pressure drops curves of different $\beta$ tend to coincide after about 10 seconds. At this time, if there is no leakage, it can be seen from the comparison with the previous Figure 6 that the measurement is stable after 10 seconds, indicating that after the measurement is stable, different $\beta$ has no impact on the accuracy of leakage detection, but only on the measurement time.

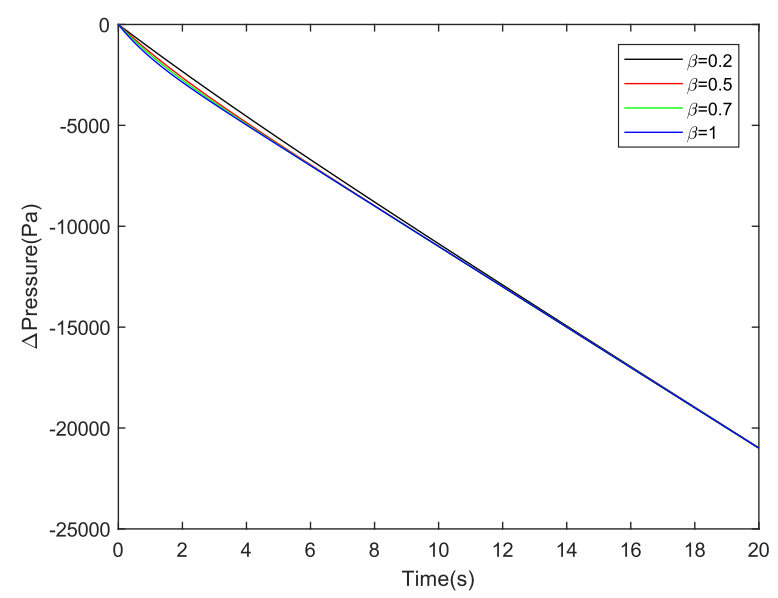

Figure 6 Relationship of $\beta$ and $\Delta p$ with leakage

Exponential parameter $\alpha$ represents the difference between the initial pressure $p_{0}$ and the final pressure $p_{\infty}$. When there is no leakage and the gas tank with general heat transfer effect is selected, when $\lambda$ is 0 and $\beta$ is 0.5 , the simulation results of $\Delta p$ and time of different $\alpha$ are shown in the Figure 7. 


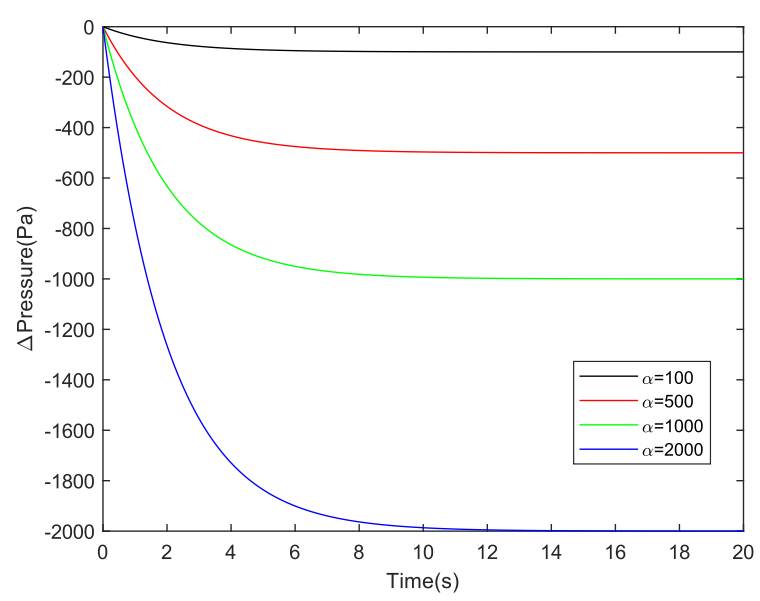

Figure 7 Relationship of $\alpha$ and $\Delta p$ without leakage

It can be seen that the overall trend of different $\alpha$ is to sharply reduce for a period of time, and then slow down the slope until it tends to their $\alpha$ value. When $\lambda$ and $\beta$ are determined, the sharp reduction time and slowdown time are the same, indicating that the measured equilibrium time is independent of the $\alpha$ value, which means the measured equilibrium time is independent of the difference between the initial pressure and the final pressure. For example, in this simulation example, the $\alpha$ is taken between $100 \mathrm{kPa}$ and $2000 \mathrm{kPa}$, and the equilibrium time is 10 to 12 seconds. At the same time, with the increase of $\alpha$, the pressure difference decreases with a large slope, indicating that for the non-leakage chamber, the temperature after inflation balance and ambient temperature have a severe impact on the measured pressure drop, which has a direct impact on the calculation of leakage, but has no impact on the measurement time.

When there is leakage and exploring the influence of $\Delta p$ of different $\alpha$, the parameter $\lambda$ is set to $1 \mathrm{kPa}$ and $\beta$ is 0.5 . The simulation results of $\Delta p$ and time of different $\alpha$ are shown in the Figure 8.

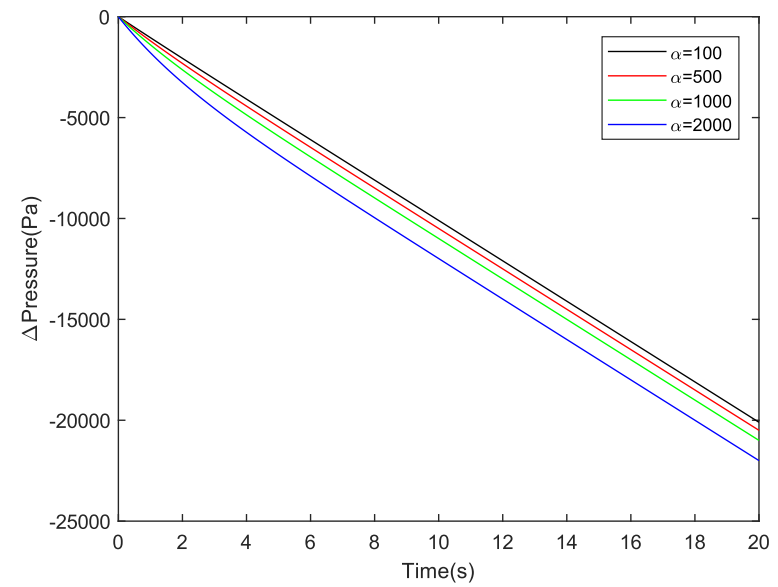

Figure 8 Relationship of $\alpha$ and $\Delta p$ with leakage

As can be seen from the Figure 8, the pressure difference of different $\alpha$ values tend to decrease, and the slope hardly changes with time. With the increase of $\alpha$, the slope of pressure drop increases continuously, and due to the effect of leakage, the pressure in the chamber will decrease until the leakage ends and the pressure in the chamber reaches atmospheric pressure.

Thus, $\alpha$ is not a characteristic parameter of the tank, but a process variable related to the detection process. When different detection pressures are taken, their values change greatly. This characteristic increases the difficulty of identification in actual measurement. In order to ensure the identification accuracy, it can first be identified the tank model $\beta$ value, further determination $\alpha$ value.

In order to determine the influence of leakage parameters on pressure drop, it is necessary to fix the inflation detection process variable $\alpha$ and the tank characteristics variable $\beta$. When $\alpha$ is $1 \mathrm{kPa}$ and $\beta$ is 0.5 , the simulation results of $\Delta p$ with different $\lambda$ in each time are shown in the Figure 9.

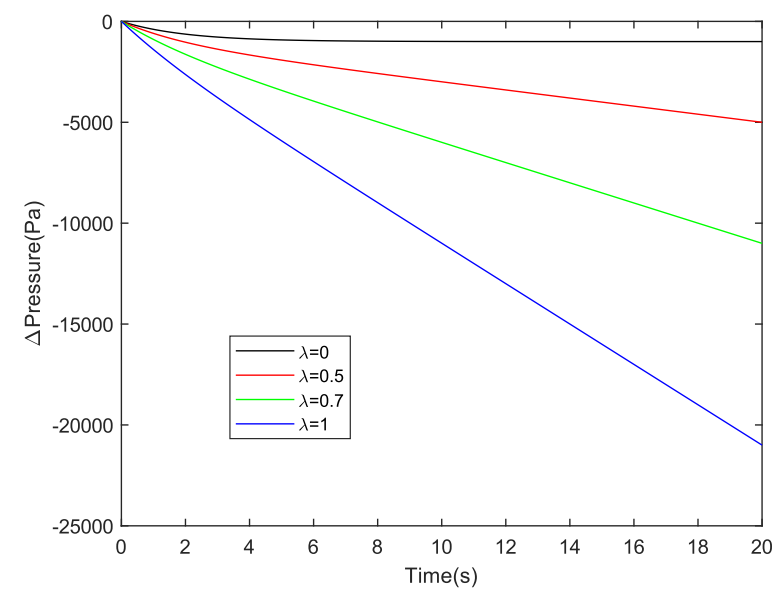


Figure 9 Relationship of $\lambda$ and $\Delta p$

This simulation compares the relationship between pressure difference and time when $\lambda$ is from 0 to 1 . It can be seen that when $\lambda$ is 0 , the pressure difference first decreases to about $4 \mathrm{~s}$, and then tends to be stable at $-1 \mathrm{kPa}$. When $\lambda$ is over 0 , the overall trend of pressure difference is always decreasing, and there is no stable value. With the increase of $\lambda$, the decreasing slope of pressure difference is also steep. $\lambda$ 's derivation formula includes not only the volume of the tested tank, but also the leakage. In the identification process, it should be analyzed according to different tanks and different leakage conditions.

Therefore, $\beta$ is relatively stable in the identification process. When the initial pressure is fixed, this value is only related to the tank itself, such as tank volume, heat transfer material and heat transfer area. Therefore, this value is easy to identify. The empirical value measured in the past or before delivery can be selected, and can be used as the characteristic parameter of the tank to characterize the heat exchange capacity of the tank. The $\alpha$ value is sensitive to the charging process. There is no empirical value for reference, nor can it be used as the characteristic parameter of the tested tank. It should be identified after $\beta$. For leakage parameters, $\lambda$ is not only related to the gas tank itself, but also related to the actual leakage, which should be identified finally.

\section{Experiment of Asymmetric Differential Pressure Method}

In order to further test the effectiveness of the pressure difference substitute formula proposed above, for nromal types of tank, the differential pressure signals during charging and measurement are collected through the data acquisition card for data analysis and identification.

\subsection{Introduction of Experimental Equipment}

The system used in the experiment is shown in the Figure 10. It uses air compressor, air tank, flow sensor, pressure sensor, temperature sensor, acquisition equipment and upper computer, and uses flow proportional valve to provide leakage. Among them, $3 \mathrm{~L}$ is used for the gas tank to be tested, 20L is used for the master gas tank, HFT-800 sensor provided by ECOSO company is used for the flow sensor, platinum wire in the range of 0 to $50{ }^{\circ} \mathrm{C}$ is used as the thermistor for the temperature sensor, and the corresponding resistance value is collected with the bridge and converted into voltage signal. The voltage signal of each sensor is calculated as the actual physical value in the upper computer through the acquisition board USB6001 of NI company. The proportional valve uses FESTO-MYPE series three position five-way voltage control valve. The valve has a position control valve core, which can convert the analog input signal into the corresponding opening size of the valve output port. The pressure sensor uses the PSE560 series of SMC company. The tank model selected in the experiment is a small one for daily use, which is highly representative.

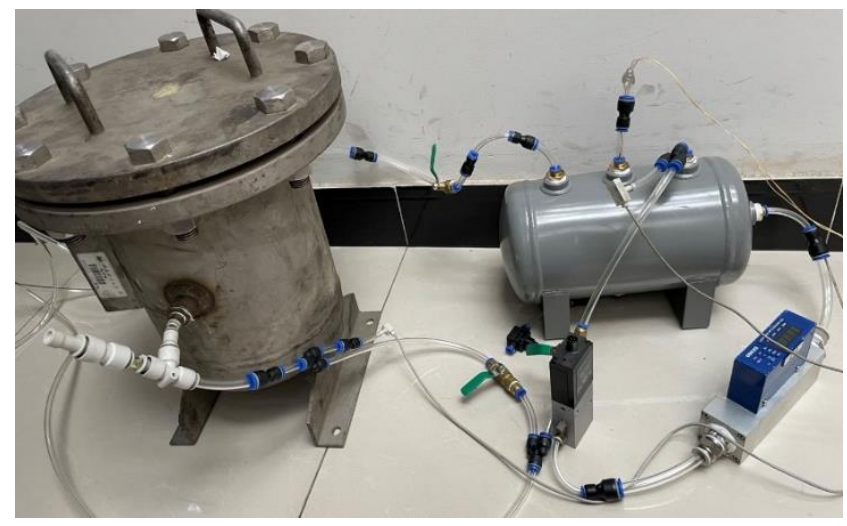

Figure 10 Experimental picture

\subsection{Experiment and Data Processing Process}

During the experiment, the valve between the air tank and the air compressor is firstly opened for charging, then the valve connecting the two air tanks is opened to balance the pressure in the two air tanks for a while and later closed. The measurement results can be used for leakage calculation.

In order to more accurately represent the measurement results and suppress the influence of random error, the original pressure data is processed by sliding smoothing in the process of data analysis, that is, the original pressure non-stationary data is regarded as nearly stable in appropriate sections and part averaged, so as to reduce the random fluctuation caused by random error. In this way, continuous local averaging between sections along the full-length data can obtain smoother measurement results and filter out the random error of frequent fluctuation.

The least square fitting is used in the fitting process. The least square method minimizes the variance between sample point $\mathrm{y}$ and function $\mathrm{f}(\mathrm{x})$ by optimizing function $\mathrm{f}$ (x), which expressed as $\min \|y-f(x)\|_{2}$. The parameters of the function can be easily identified by the least square method. In this experiment, the smoothed data are fitted by least squares and determine $\alpha, \beta, \lambda$. Finally, after replacing the differential pressure affected by temperature, whether the leakage value is consistent with the leakage of the actual tank is analyzed. 


\subsection{Cavity Leakage Detection Results}

The $3 \mathrm{~L}$ gas tank is used as the gas tank to be tested, and the collected field data is pretreated with sliding smoothing, as shown in the Figure 11 and Figure 12. In the charging stage, because the maximum amount is much greater than the leakage, the pressure of the two chambers increases synchronously. After reaching about 5bar at 200s, the air source is closed, and the valve between the two chambers is opened to balance for a period of time. The pressure of the tested chamber decreases sharply due to leakage, while the pressure of the master chamber decreases slowly due to heat dissipation. This makes the differential pressure have no obvious amplitude in the charging phase, but reduce to 4 bar in the measurement phase.

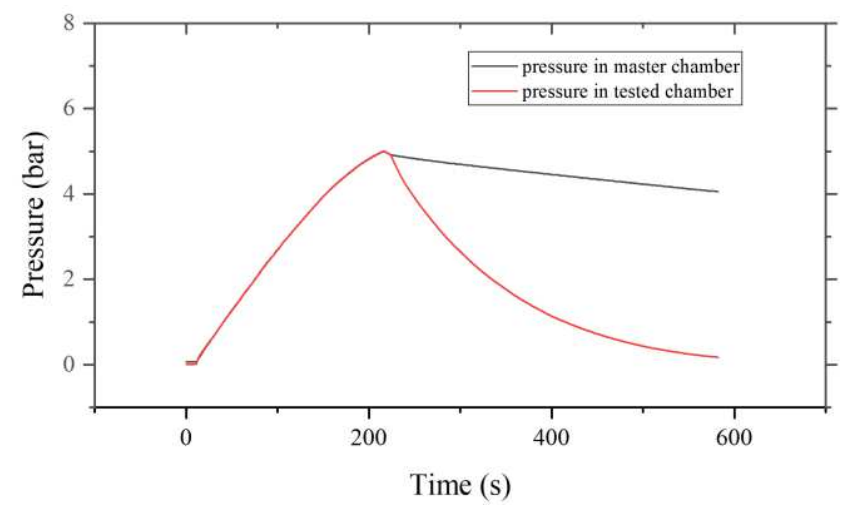

Figure 11 Pressure variation of two chambers with time

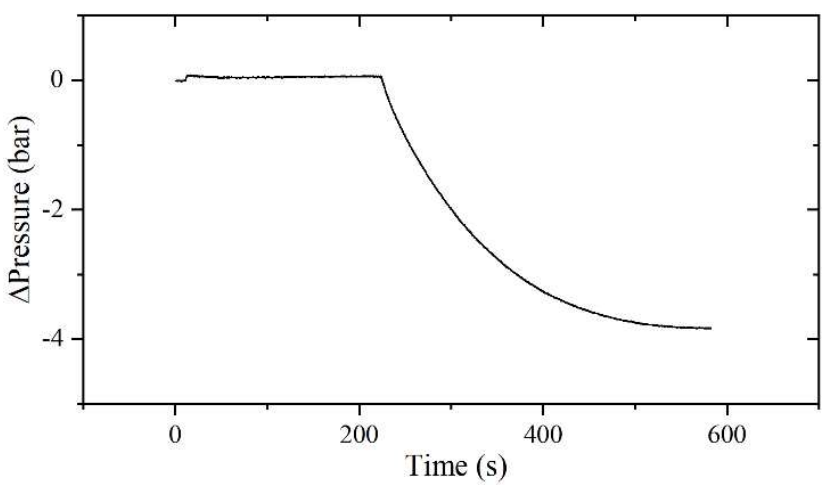

Figure 12 Variation of pressure difference with time

The pressure difference in the measurement stage is selected separately for exponential function fitting. Within 95\% confidence bounds, it is calculated as Table 1 .

Table 1 Parameter fitting results

\begin{tabular}{ccc}
\hline$\alpha$ & $\beta$ & $\lambda$ \\
\hline 4.667 & 0.008115 & 0.001567 \\
\hline
\end{tabular}

And get the goodness of fit as follows in Table 2.
Table 2 Parameter fitting effect

\begin{tabular}{ccc}
\hline SSE & R-square & RMSE \\
\hline 0.4808 & 0.9996 & 0.02119 \\
\hline
\end{tabular}

In the charging stage, the temperature increases first and then decreases slowly, which is due to the rapid compression of air in the tank during charging and the increase of molecular heat movement, resulting in the rise of temperature. With the slowdown of charging, the heat transfer system of the tank begins to dissipate heat to the environment, resulting in the decrease of temperature in the later stage of inflation stage. During the measurement stage, the temperature continues to decrease due to leakage. The temperature characteristic data of the two stages are shown in the Table 3.

Table 3 Temperature characteristic data of two stages

\begin{tabular}{ccc}
\hline & Charging stage & Measurement stage \\
\hline Maximum value & $25.67^{\circ} \mathrm{C}$ & $25.48^{\circ} \mathrm{C}$ \\
Average value & $25.55^{\circ} \mathrm{C}$ & $25.26^{\circ} \mathrm{C}$ \\
Minimum value & $25.43^{\circ} \mathrm{C}$ & $25.03^{\circ} \mathrm{C}$ \\
\hline
\end{tabular}

Comparing leakage calculation between conventional method and substitute formula method, it can be seen in Figure 13 that the fitting result has good effect and can converge to the correct leakage interval without fluctuation. In the early stage of measurement, the calculated value of low leakage is about $7.5 \mathrm{~L} / \mathrm{min}$, while the leakage calculated by differential pressure method is about 9.5 $\mathrm{L} / \mathrm{min}$, and there is a maximum oscillation deviation of 0.5 $\mathrm{L} / \mathrm{min}$ during the measurement process.

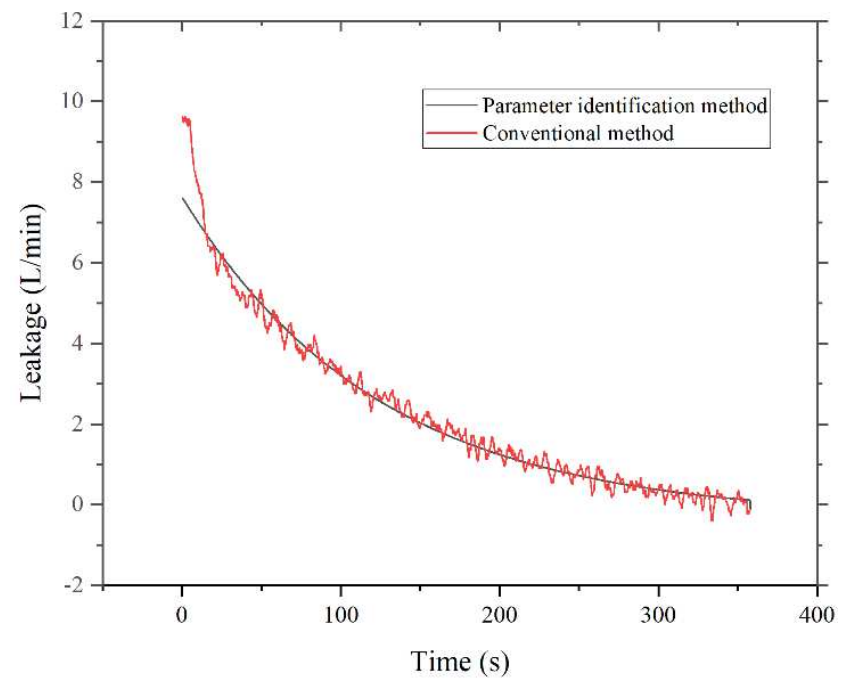

Figure 13 Comparison of leakage calculation between conventional method and substitute formula method 
In Figure 13, it can be seen from the experiment that using the differential pressure substitute formula to calculate the leakage can obtain a more accurate value without large vibration, and some parameters in the formula can be obtained from experience, and some parameters can be obtained by controlling charging or measurement methods, which has highly derivability.

\section{Conclusion}

In this paper, the pressure difference is fitted into an exponential formula to replace the differential calculation of pressure difference in conventional leakage detection. It has the characteristics of small fluctuation, intuitive measurement and fast measurement speed. In the exponential formula, the characteristic parameter of the chamber, the measurement process parameter and the leakage characteristic parameter will affect the value of the pressure difference in the substitute formula at each time point. The influence of the three parameters on the pressure difference and the influence of the actual physical parameters affecting the three parameters on the pressure difference are explored through simulation, and the sequence scheme of identifying the parameters is obtained. Finally, the superiority of the pressure difference fitting formula is verified by experiments.

\section{Declaration}

\section{Acknowledgements}

Not applicable

\section{Funding}

Supported by National Key R\&D Program of China (Grant No. 2019YFC0121702) and National Key R\&D Program of China (Grant No. 2019YFC0121703).

\section{Competing interests}

The authors declare no competing financial interests.

\section{Availability of data and materials}

The datasets supporting the conclusions of this article are included within the article.

\section{Authors' contributions}

The author' contributions are as follows: Yan Shi and Xue-Lin Zhao are responsible for the conception of the theory, Jia-Qi Chang is responsible for building the experimental platform and collecting data, Yi-Xuan Wang is responsible for the writing of the article, and the other authors are responsible for the revision of the article.

\section{Competing interests}

The authors declare no competing financial interests.

\section{Consent for publication}

Not applicable

\section{Ethics approval and consent to participate}

Not applicable

\section{References}

[1] Shirato Y, Ohnishi W, Fujimoto H, et al. Controller design of mass flow rate loop for high-precision pneumatic actuator. 16th International Workshop on Advanced Motion Control (AMC),2020.

[2] Ho Cong Minh and Tran Duc Thien and Ahn Kyoung Kwan. Adaptive sliding mode control based nonlinear disturbance observer for active suspension with pneumatic spring. Journal of Sound and Vibration, 2021,509.

[3] Haraguchi, D, et al. A Pneumatically Driven Surgical Manipulator With a Flexible Distal Joint Capable of Force Sensing. IEEE/ASME Transactions on Mechatronics, 2015,20(6):2950-2961.

[4] X. Liu, J. Liu and S. Li, Parameter modification model of fluid flow rate for a pneumatically-actuated PDMS membrane microvalve. 2015 International Conference on Fluid Power and Mechatronics (FPM),2015: 700-705.

[5] Tao W, Wang X, Bo W, et al. Gas leak location method based on annular ultrasonic sensor array. 2018 IEEE International Instrumentation and Measurement Technology Conference (I2MTC), 2018.

[6] Wang D, Zhao F, Tao W. The ultrasonic characteristics study of weak gas leakage. 2015 International Conference on Fluid Power and Mechatronics (FPM),2015.

[7] Piazzetta G R, Flesch R, Pacheco A. Leak Detection in Pressure Vessels Using Ultrasonic Techniques. ASME 2017 Pressure Vessels and Piping Conference,2017.

[8] Ling Z, Tao W, Peng S, et al. A novel adaptive leak diagnosis and localization method for infrared image. International Journal of Innovative Computing Information \& Control, 2012, 8(5B):3553-3563.

[9] Daniels C C, Braun M J, Oravec H A, et al. Leak Rate Quantification Method for Gas Pressure Seals with Controlled Pressure Differential. Journal of Spacecraft and Rockets, 2017,54(6):1228-1234.

[10]Guntur H L, Cai M, Kawashima K, et al. Analysis of Temperature Effect on Differential Pressure Method for Air Leak Detection.SICE Conference, 2004.

[11]Cai, M. , Kawashima, K. , \& Kagawa, T. . Characteristics of leak detection based on diferential pressure measurement. Symposium on Fluid Power, 2005(6).

[12]Harus L G, Cai M, Kawashima K, et al. Determination of temperature recovery time in differential-pressure-based air leak detector. Measurement Science \& Technology, 2006, 17(2):411-418.

[13]Harus L G, Cai M, Kawashima K, et al. The Effect of Temperature Recovery Time on the Repeatability of a Differential Pressure Type Air Leak Tester. Transactions of the Japan Hydraulics \& Pneumatics Society, 2007, 38(4):54-59. 
[14]Feng Jian and Zhang Huaguang, Oil pipeline leak detection and location using double sensors pressure gradient method. Fifth World Congress on Intelligent Control and Automation, 2004, 4: 3134-3137.

[15]Chen S, Yan S, Wang L, et al. Development on Dynamic Pressure Monitoring Method and Sensor for Long Pipeline Leak Detection. International Pipeline Conference. 2008.

\section{Biographical notes}

Yan Shi, born in 1981, is currently a professor at School of Automation Science and Electrical Engineering, Beihang University, China.

E-mail: shiyan@buaa.edu.cn

Jia-Qi Chang, born in 1996, is currently a PhD candidate at School of Automation Science and Electrical Engineering, Beihang University, China.

E-mail: chang_jiaqi@buaa.edu.cn

Yi-Xuan Wang, born in 1989, is currently a lecturer at School of Automation Science and Electrical Engineering, Beihang University, China.

E-mail: magic_wyx@163.com

Xue-Lin Zhao, works currently at Department of Musculoskeletal tumor, Senior Department of Orthopedics, the Fourth Medical Center of PLA General Hospital, China.

E-mail: zxl_199013@163.com

Qing-Zhen Zhang, is currently an associate professor at School of Automation Science and Electrical Engineering, Beihang University, China.

E-mail: zhangqz@buaa.edu.cn

Li-Man Yang, is currently an associate professor at School of Automation Science and Electrical Engineering, Beihang University, China.

E-mail: ylm@buaa.edu.cn 